\title{
Uso de Nuevos Recursos Tecnológicos en la Docencia de un Curso de Anatomía con Orientación Clínica para Estudiantes de Medicina
}

\author{
Use of New Technological Resources in the Teaching \\ of a Clinical Anatomy Course for Medicine Students
}

Pedro Hecht López \& Andrea Larrazábal Miranda

HECHT, L. P. \& LARRAZÁBAL, M.A. Uso de nuevos recursos tecnológicos en la docencia de un curso de anatomía con orientación clínica para alumnos de medicina. Int. J. Morphol., 36(3):821-828, 2018.

RESUMEN: El uso de recursos tecnológicos como material de apoyo en la docencia de anatomía humana tiene antecedentes históricos en Chile desde el siglo 19, con la llegada del primer modelo anatómico artificial. En conjunto con las tradicionales clases magistrales y lectura de los textos clásicos sobre la materia, durante los trabajos prácticos se presentaba al estudiante una serie de contenidos estrictamente anatómicos que posteriormente serían evaluados. Esta metodología de enseñanza se mantuvo hasta muy avanzado el siglo 20. Posteriormente con los avances en tecnología médica de las últimas décadas incorporados a la práctica clínica habitual, también debieron integrarse conocimientos sobre las estructuras anatómicas, en cuanto a su representación en los exámenes obtenidos mediante técnicas de imágenes médicas. Por esta razón para la formación de las nuevas generaciones de médicos, se han debido actualizar paulatinamente; la literatura básica integrando a ella contenidos clínicos, la metodología docente haciéndola participativa hacia el estudiante y en el último tiempo los materiales para trabajos prácticos, de manera que se logre un aprendizaje duradero, cuyos contenidos sean utilizados posteriormente en otras asignaturas básicas y clínicas. Previo a iniciar las actividades docentes en el laboratorio de anatomía de la nueva Escuela de Medicina de la Universidad de Tarapacá, Chile, la institución adquirió material de apoyo docente de última generación para complementar la enseñanza en los trabajos prácticos de anatomía. En este artículo se describirá la implementación de los nuevos recursos tecnológicos de apoyo docente en anatomía para nuestra escuela de medicina, entre los cuales se encuentran: modelos anatómicos impresos en tres dimensiones (3D) Erler-Zimmer®, cadáver humano sintético Syndaver®, mesa de disección digital 3D SECTRA® y su estación de trabajo complementaria. Todos estos materiales se unieron al uso de otros recursos tradicionales en la enseñanza de esta disciplina, para entregar los contenidos de la anatomía con un enfoque combinado.

PALABRAS CLAVE: Impresión 3D; Modelo anatómico; Disección digital; Cadáver sintético; Educación médica.

\section{INTRODUCCIÓN}

El estudio de la anatomía humana tiene como uno de sus primeros referentes a las prácticas de disección cadavérica realizadas en el antiguo Egipto según consta en el registro del papiro de Hadest con data de 1500 años a.C. Por otra parte en Alejandría y antigua Grecia se realizó la disección cadavérica por Herófilo de Calcedonia y Erasístrato hacia el siglo 3 a.C.

Pero el inicio de la anatomía moderna y su estudio se remonta a los trabajos de Andreas Vesalio y la publicación de su obra "De humani corporis fabrica" en 1542. Este último autor innovó el sistema didáctico entre maestro y alumnos, al enseñar la anatomía combinando el uso del cadáver, libros y dibujos para una mejor explicación. (Montemayor, 2006).
Actualmente el acceso a material cadavérico con fines docentes se ha visto afectado, lo cual ha impactado en la enseñanza de la disciplina (Inzunza et al., 2015). Esto último es observado en el medio local debido a distintas causas, situación que también se hace presente en la realidad educativa de otros países.

Este fenómeno de escases de materiales no ha sido un evento aislado en el tiempo, ya que, en diferentes épocas de la historia han existido limitaciones por causas religiosas, éticas y legales por mencionar algunas. Para paliar el déficit y lograr una enseñanza efectiva, en el transcurso de la historia las explicaciones teóricas se han apoyado con ilustraciones de distinto grado de detalle, muchas de ellas ex- 
quisitamente reproducidas a partir de la observación de una pieza anatómica. Estas ilustraciones vienen a complementar lo detallado en los tratados de la materia.

Considerando lo anterior, se ha reportado que en nuestro país existe un antiguo precedente de la enseñanza de anatomía con innovación a través de tecnología. Ya en el año 1864 se apoya la docencia mediante la estatua anatómica del Dr. Auzoux, considerada el primer modelo anatómico de uso docente en Chile. Esta corresponde a una estatua que representa a un hombre adulto en posición de pie, sin su piel, exponiendo su musculatura y otros detalles anatómicos. De aproximadamente 1,80 metros de altura, fue fabricada con papel maché y pintada al óleo en Francia. Compuesta de partes articuladas por sistemas de ganchos y argollas, se encuentran en ella 92 partes y 2000 detalles identificados con un número. Este modelo anatómico sirvió a varias generaciones de estudiantes hasta los primeros decenios del siglo 20 (Cárdenas, 2015).

En cuanto a la metodología docente, hasta el inicio del nuevo milenio inclusive, la enseñanza de la medicina (de la cual anatomía es un pilar importante durante el primer año de la carrera), se ha realizado de la manera tradicional centrada en el profesor y basada en la entrega de información de un conocimiento disciplinar aislado. En este modelo la asignatura se encuentra situada dentro de una malla curricular rígida, donde el aprendizaje se logra mediante la oportunidad de estudiar un tema específico disponible (Lermanda, 2007).

Por otra parte debemos consignar que nuestra novel escuela de medicina, se encuentra inserta en la realidad de una provincia, en el extremo norte del país a más de $2000 \mathrm{~km}$ de la capital, integrándose a una universidad estatal donde el nuevo modelo educativo promueve un proceso enseñanza-aprendizaje centrado en el estudiante, valiéndose de estrategias didácticas apoyadas con las tecnologías de la información y comunicación (TIC). En este contexto, la autoridad universitaria luego de revisar el estado del arte de los materiales didácticos en anatomía decide implementar nuestro laboratorio con recursos tecnológicos de apoyo docente de última generación, entre los cuales se encuentran: modelos anatómicos impresos 3D Erler-Zimmer® de procedencia Alemana, un cadáver humano sintético Syndaver® fabricado en los Estados Unidos de Norteamérica, además, una mesa de disección digital 3D SECTRA $®$ de procedencia europea y su estación de trabajo complementaria, las cuales poseen el programa $\mathrm{VH}$ Dissector ${ }^{\circledR}$ con las herramientas para estudiar la anatomía de un cadáver humano real digitalizado. Este último equipo informático, además, está enlazado vía internet con una base de datos en plataforma IDS7 desarrollada por el fabricante SECTRA ${ }^{\circledast}$, de ella una parte está alojada en un servidor local del laboratorio y otra parte en un servidor de esa empresa en Dublin. Mediante esta base de datos se puede acceder a una serie de casos de anatomía con orientación clínica recopilados con fines de docencia, los cuales fueron realizados y compartidos por instituciones de educación superior de diferentes países.

Todos estos nuevos materiales se unieron al uso de otros recursos actualmente usados para la enseñanza de esta disciplina en las carreras de medicina de otras Universidades, tales como las clases interactivas, lecturas dirigidas, clase invertida, aprendizaje basado en problemas o casos (ABP) y el estudio de la osteología humana mediante la colección de huesos del laboratorio. El conjunto de recursos anteriores se implementó para entregar los contenidos de la anatomía con un enfoque combinado. Con esta metodología se persigue integrar la anatomía con orientación clínica y las nuevas tecnologías para lograr un aprendizaje significativo de los contenidos perdurando en el tiempo.

En este trabajo se describirá nuestra experiencia en la implementación del laboratorio de anatomía de una nueva escuela de medicina usando principalmente recursos tecnológicos de última generación con fines docentes.

\section{MATERIAL Y MÉTODO}

\section{Recursos Tradicionales}

Colección de huesos humanos. Este trabajo se refiere en su mayor parte al proceso de implementación de recursos tecnológicos modernos en la disciplina de anatomía, no obstante se debe declarar que para las actividades de laboratorio referentes a osteología, durante los meses previos al inicio de labores docentes, se preparó una serie de 15 cuerpos humanos en estado de osamentas, las cuales fueron obtenidas mediante un convenio de cooperación entre el cementerio municipal local y nuestra universidad. La mayor parte de estos huesos se encontraban con un grado de conservación suficiente para servir en la enseñanza de la osteología. Esta colección de huesos pasó a formar parte de los recursos que tradicionalmente se han usado en el aprendizaje de la anatomía en las carreras de medicina. Una parte de ellos se dispuso en el laboratorio docente para su fácil acceso durante las actividades prácticas, la otra parte se dejó disponible en la bodega de anatomía para el préstamo a los alumnos que lo requirieran en su estudio personal, durante los horarios fuera de actividades presenciales obligatorias de la asignatura. 
Estudio de textos guía. Para que los alumnos complementaran el estudio de los temas expuestos en las clases, se implementó la biblioteca del campus con las últimas ediciones de textos tipo tratado de anatomía humana con orientación clínica, además, un número adecuado de libros tipo atlas de ilustraciones anatómicas y atlas fotográfico de anatomía cadavérica. Todos estos recursos se pusieron a disposición de los estudiantes mediante el sistema informático de préstamos de libros de la biblioteca de nuestra Universidad.

Guías de laboratorio. El estudio personal y preparación de los pasos prácticos de anatomía, fue realizado por los alumnos mediante el estudio previo de los temas ocupando los textos antes descritos y el desarrollo de una serie de guías de laboratorio entregadas días antes de cada actividad. En estas mediante una introducción se orientaba al alumno acerca del tema a desarrollar durante la sesión y se entregaba una serie de láminas para practicar el rotulado de estructuras anatómicas, las cuales en su parte final disponían de un solucionario para cotejar las respuestas. También se daba una serie de ejercicios propuestos que incluían el desarrollo de tablas resumen y glosarios sobre términos anatómicos con relevancia clínica. Por último en cada guía se dio una pauta de trabajo para la prospección de los contenidos prácticos a revisar en cada mesa, ocupando los materiales dispuestos en el laboratorio.

\section{Nuevos recursos tecnológicos}

Impresiones 3D. Para las actividades prácticas en el laboratorio y revisión de los contenidos homologables a la prospección de preparados anatómicos de cadáveres, se dispuso de una serie de modelos anatómicos impresos en 3D copia fiel de segmentos anatómicos de cadáveres disecados, confeccionados por la empresa Erler-Zimmer ${ }^{\circledR}$ en Alemania. Los impresos 3D permitieron exponer los detalles anatómicos de partes blandas más pequeñas como por ejemplo en las regiones de cabeza, cuello y extremidades, que fueran difíciles de observar o presentar en otros materiales innovadores (Fig. 1). Este tipo de impresiones permitió subsanar la falta de material cadavérico fresco y el importante gasto en recursos derivados de su preparación y conservación en cámaras de frío y un anfiteatro dedicado a este fin.

Cadáver humano sintético. La anatomía macroscópica humana se presentó también mediante el uso de un fantoma cadáver humano sintético Syndaver ${ }^{\circledR}$. Este recurso fue fabricado por la empresa SyndaverLabs ${ }^{\circledR}$ en Estados Unidos de Norteamérica (EUA) y sirvió para estudiar las estructuras anatómicas más gruesas, distintos órganos y sus relaciones en las diferentes profundidades del cuerpo humano completo (Fig. 2). Además, debido a lo innovador de los materiales usados en su confección, este modelo anatómico permitió a los estudiantes conocer la textura y propiedades de los tejidos en un estado similar al sujeto vivo, a diferencia de lo que sucede en los clásicos preparados de cadáveres fijados en formalina o sometidos a plastinación ocupados en otros laboratorios, donde estos procesos químicos modifican en forma importante la textura y color de los tejidos en fresco.

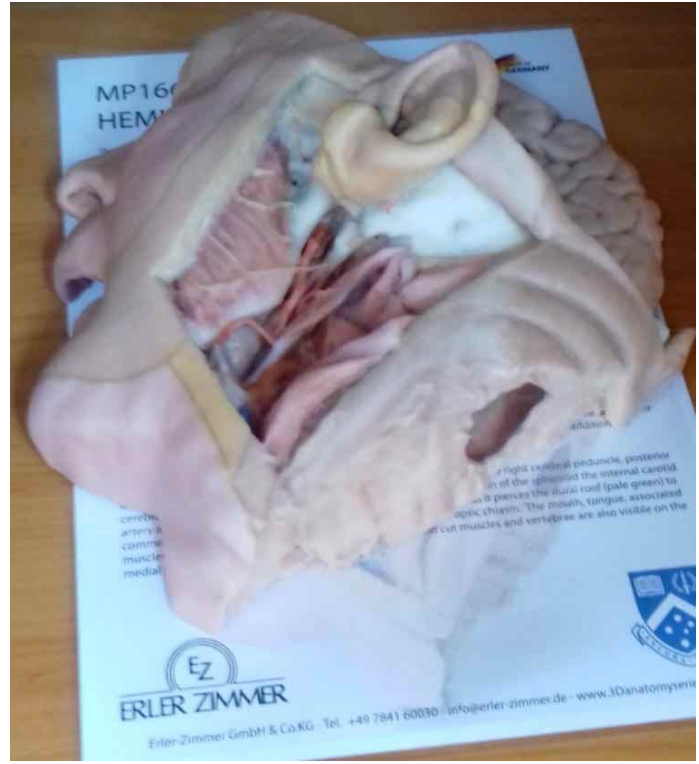

Fig. 1. Modelo anatómico de las regiones cabeza y cue1lo. Impresión 3D Erler-Zimmer® obtenida por el fabricante a partir de una disección cadavérica.

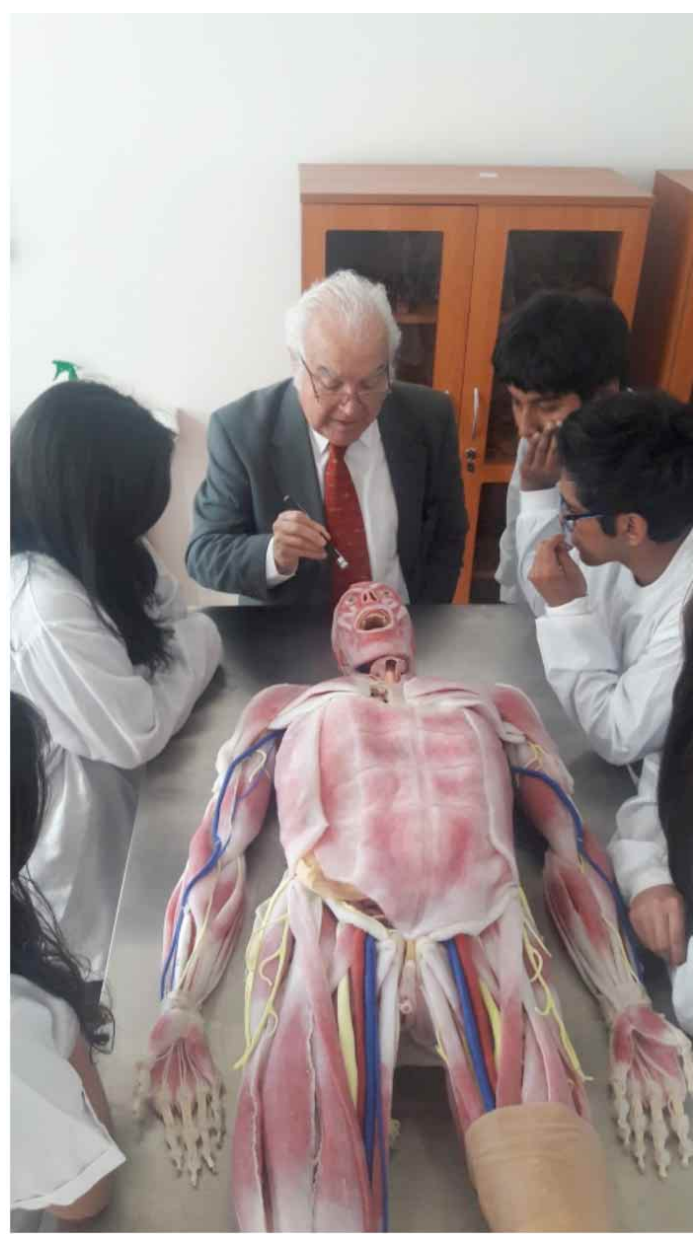

Fig. 2. Cadáver humano sintético Syndaver® y su uso docente en el laboratorio de anatomía. 
Mesa de disección digital 3D. Para el ejercicio de prospección y visualización de diferentes estructuras anatómicas desde el punto de vista topográfico, se dispuso de la representación digitalizada de un cadáver humano real proveniente de la base de datos del "proyecto humano visible" (Visible Human Project ${ }^{\circledR}$, National Library of Medicine). Estas imágenes digitales se trabajaron usando el programa $\mathrm{VH}$ Dissector ${ }^{\circledR}$ instalado en una mesa de visualización digital 3D táctil del fabricante SECTRA® con procedencia Europea. El trabajo en este equipo, permitió realizar la disección digital y separación por capas de las estructuras anatómicas de interés para las diferentes unidades de estudio en la asignatura. Además se pudo realizar el estudio mediante cortes en diferentes planos anatómicos y reconstrucción 3D de los distintos segmentos (Fig. 3). Esto último fue muy importante para la integración de conocimientos sobre la anatomía seccional y su correlación con las imágenes obtenidas mediante las diferentes técnicas de imagenología moderna, tales como tomografía computada (TC) o resonancia magnética $(\mathrm{RM})$. Las imágenes médicas se trabajaron mediante la plataforma informática IDS7, cuya base de datos en su mayor parte fue proporcionada por el desarrollador SECTRA®, este programa permitió manipular y visualizar las imágenes digitales tanto en un plano, como realizar reconstrucciones multiplanares (RMP) y de volúmenes en 3D.

Estación de trabajo 3D complementaria. Con el fin de facilitar el proceso de aprendizaje de los alumnos mediante grupos pequeños durante el desarrollo de los trabajos prácticos, se implementó una pantalla táctil adicional para trabajar en forma paralela y complementaria a la mesa de disección digital antes descrita. En esta pantalla se instaló el programa VH

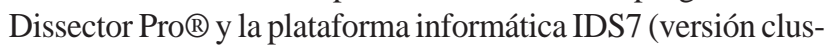
ter) disponiéndose de conexión vía internet a la misma base de datos que en la mesa de disección principal. Este recurso se dispuso en el laboratorio para que los alumnos pudieran guiarse durante la prospección de los diferentes modelos anatómicos, así como también revisar la anatomía radiológica de los segmentos estudiados. La misma pantalla funciona también como estación de trabajo, ya que, dispone de un potente procesador y acelerador gráfico 3D con la capacidad para segmentar, reconstruir y presentar nuevos casos locales de anatomía radiológica, obtenidos a partir de exámenes con datos en formato DICOM.

Encuesta de percepción sobre la docencia en anatomía. Luego de finalizar las actividades obligatorias anuales de los pasos prácticos de anatomía y con el objetivo de evaluar la percepción de los alumnos con respecto a la calidad e implementación de la asignatura, se aplicó al grupo de estudiantes una encuesta de tipo voluntaria, auto-administrada y anónima. Para el actual reporte se priorizaron los datos obtenidos a partir de preguntas referentes a nuevas tecnologías y metodologías, cuyas respuestas corresponden a la selección de alternativas en distintos grados de acuerdo o desacuerdo con respecto a la pregunta. En la administración de este instrumento se incluyó a todos los alumnos que completaran el total de las actividades prácticas y evaluaciones finalizado el segundo semestre del curso. Del número inicial de alumnos matriculados en el curso $(n=30)$ debemos declarar que 29 calificaron para la administración de la encuesta, de este último grupo todos aceptaron completar el instrumento de medición. El único alumno que no se incluyó en la encuesta, corresponde a un reprobado al final del primer semestre quien no pudo continuar desarrollando las actividades prácticas de la segunda parte de la asignatura por no contar con el pre-requisito aprobado.

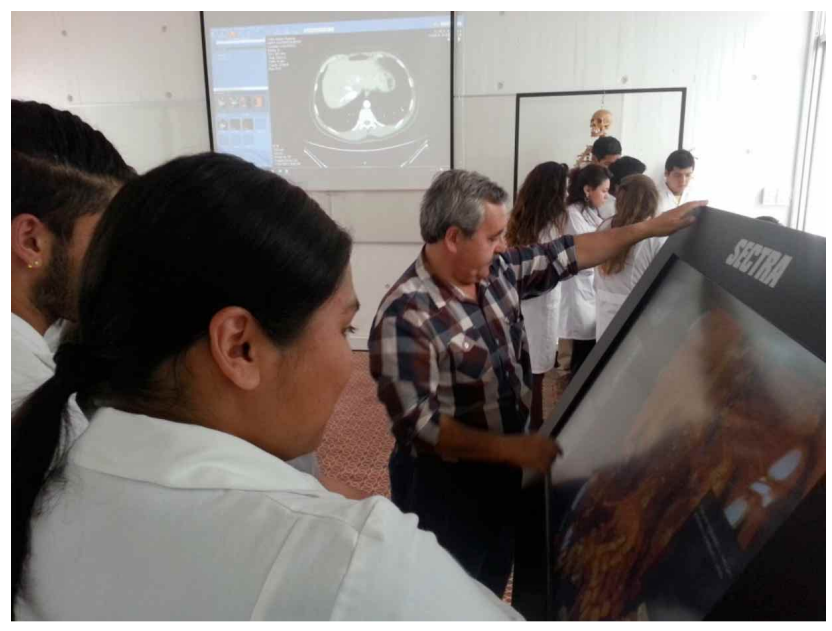

Fig. 3. Mesa táctil de visualización digital 3D SECTRA® en uso durante las actividades prácticas del laboratorio de anatomía

\section{RESULTADOS}

Primera cohorte de alumnos de medicina. Debido a que la implementación de nuevos recursos tecnológicos actualizados para la enseñanza de anatomía humana, no tiene un precedente similar en las carreras de pregrado del medio local, los resultados obtenidos deben ser analizados tomando en cuenta que son la línea de base de esta asignatura que inició sus labores al nacer una nueva escuela de medicina y donde la cohorte de alumnos de la primera generación se puede considerar una pequeña muestra, en comparación con la gran capacidad de matrícula de otras escuelas de medicina en nuestro país. Por otra parte se debe considerar que nuestros estudiantes durante su enseñanza media fueron de alto rendimiento académico y lograron buenos puntajes de ingreso a esta carrera de la Facultad de Ciencias de la Salud, lo que puede orientarnos sobre sus hábitos y compromiso con el estudio de la asignatura. De 
los 29 alumnos 15 mujeres (52\%) y 14 hombres (48\%) completaron la encuesta. Aproximadamente el $50 \%$ del total provienen de la región de Arica y el resto declaran venir desde otras regiones, con una amplia distribución, que va desde la vecina región de Tarapacá, hasta regiones de la zona central y el sur del país. Para caracterizar el rendimiento académico del grupo que respondió las encuestas, podemos informar que al finalizar las actividades de evaluación del segundo semestre, todos calificaron con nota de aprobación para enfrentar el examen final de primera oportunidad. Al concluir el curso del segundo semestre no se registraron alumnos reprobados, pero cabe mencionar la existencia de dos alumnos con rendimiento límite los cuales necesitaron seguimiento y apoyo especial mediante el programa de tutorías para remediar su riesgo de reprobación. En este grupo, en cuanto a las calificaciones finales de la asignatura en el segundo semestre se registró una nota promedio de 5,59 con una desviación estándar de 0,59 donde la máxima nota fue 6,4 y la mínima 4,3 (con escala de 1,0 a 7,0 y nota mínima de aprobación 4,0). Con respecto al rendimiento de la parte laboratorio se debe señalar que no hubieron reprobados, lo cual permitió que todos accedieran a enfrentar el examen final. Las notas finales de laboratorio registraron un promedio de 5,55 con una desviación estándar de 0,49 donde la máxima nota fue 6,4 y la mínima 4,4 (con la misma escala de calificación).

Resultados de percepción sobre nuevas tecnologías en anatomía. Para caracterizar la percepción de los estudiantes en cuanto a la implementación de nuevos recursos tecnológicos en la enseñanza de la anatomía humana, se mostrarán las figuras obtenidas a partir de las respuestas a preguntas que tienen que ver con el uso de los nuevos materiales utilizados y su comparación con otros recursos tradicionales.

Pregunta ¿Considera que los trabajos prácticos con modelos anatómicos impresos 3D (fantomas impresos 3D de alta fidelidad Erler-Zimmer ${ }^{\circledR}$ ) facilitan el estudio de los temas o unidades en que fueron utilizados?

En la Figura 4 podemos observar que el 86,2 \% de los alumnos está de acuerdo o totalmente de acuerdo en cuanto a la utilidad de las impresiones anatómicas 3D para el estudio de la materia. Por otra parte sólo un 6,9\% tiene una opinión desfavorable acerca de este recurso.

Pregunta. ¿Considera que el uso de un cadáver humano sintético (fantoma Syndaver ${ }^{\circledR}$ ) facilita el aprendizaje de estructuras anatómicas macroscópicas (o de mayor tamaño) y las relaciones entre sistemas de órganos?

En la Figura 5 se observa que el 86,22\% de los alum-

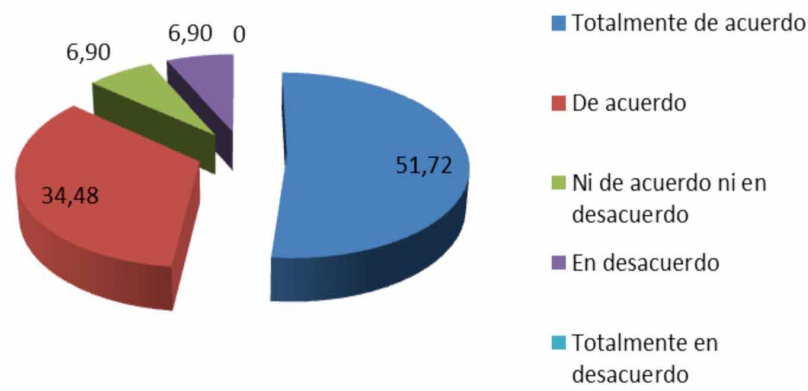

Fig. 4. Respuestas de los alumnos en relación a la utilidad de las impresiones anatómicas 3D.

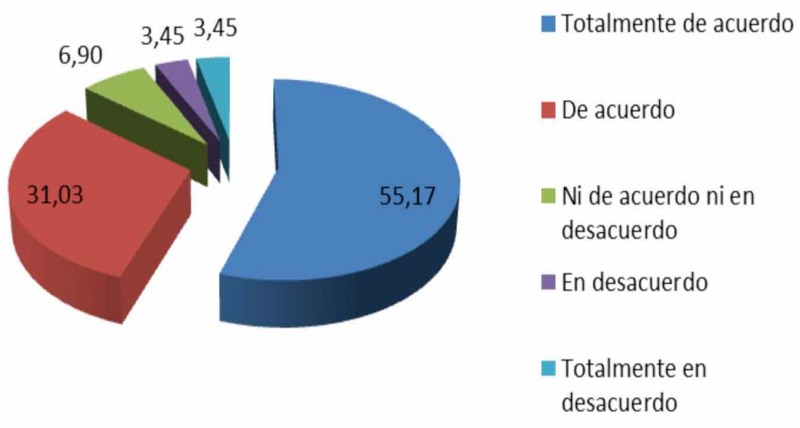

Fig. 5. Respuestas de los alumnos en relación a la utilidad del cadáver sintético Syndaver ${ }^{\circledR}$.

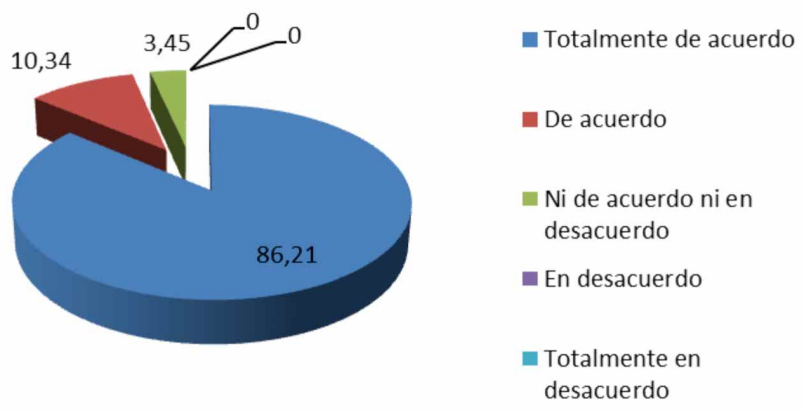

Fig. 6. Respuestas de los alumnos (\%) en relación a la utilidad de los recursos digitales en las plataformas disponibles del laboratorio en el aprendizaje de la anatomía.

nos está de acuerdo o totalmente de acuerdo en la utilidad del cadáver sintético Syndaver ${ }^{\circledR}$ para el aprendizaje de las estructuras anatómicas de mayor tamaño. Sólo el 6,9 \% de los alumnos tiene un grado de desacuerdo acerca de la utilidad de este recurso. 
Pregunta. ¿Considera que el uso de la mesa de disección digital SECTRA ${ }^{\circledR}$ y sus programas 3D (Atlas, VH Dissector ${ }^{\circledR}$, IDS7 para radiología) facilitan el estudio y aprendizaje de los contenidos del curso anatomía?

El 96,55\% de los estudiantes está de acuerdo o totalmente de acuerdo con la utilidad en el aprendizaje de la anatomía al utilizar los recursos digitales en las plataformas disponibles en nuestro laboratorio. Destacar que no existen opiniones negativas respecto a este recurso y sólo un 3,45\% de los encuestados manifestó una posición indiferente (Fig. 6).

Pregunta $i E l$ uso de recursos modernos 3D (fantomas, mesas digitales) facilita más el aprendizaje de la anatomía en comparación con recursos clásicos 2D (atlas de dibujos, atlas fotográfico, textos clásicos)?

El 93,1\% de los estudiantes respondieron que se encontraban totalmente de acuerdo y el 6,9\% de acuerdo con la mayor utilidad de los nuevos recursos tecnológicos y 3D en el aprendizaje de la anatomía en comparación con los recursos clásicos en 2D. No hubo opiniones negativas ni indiferentes con respecto a esta pregunta.

\section{DISCUSIÓN}

Durante los últimos años a nivel internacional tanto en EUA cómo en Europa, la introducción de nuevos recursos tecnológicos con fines docentes en anatomía ha tenido una progresiva implementación, inclusive en prestigiosas Universidades con histórica trayectoria en la enseñanza de anatomía mediante materiales clásicos (Turnis, 2014).

A nivel nacional en general se mantiene una metodología clásica en la entrega docente y el uso de material cadavérico durante los trabajos prácticos, existiendo algunas excepciones en escuelas de medicina que han implementado en parte recursos digitales multimedia y una que ha reportado el uso de impresión 3D para generar sus propias réplicas de secciones anatómicas de material cadavérico (Inzunza et al.).

El laboratorio de anatomía de nuestra novel escuela de medicina se implementó a partir de los recursos originados desde un proyecto conjunto entre la Universidad de Tarapacá y el Gobierno Regional de Arica y Parinacota. Este esfuerzo en una región extrema de Chile, tiene como objetivo formar médicos generales que satisfagan las grandes necesidades de prestaciones médicas en nuestro medio, valiéndose de una educación de calidad a partir del uso de innovaciones didácticas e implementación de recursos tecnológicos docentes de vanguardia, para lograr un aprendizaje significativo.
Debido a que en la etapa de proyecto los procesos de adquisición de material cadavérico para el laboratorio sufrieron considerables reparos y demoras de orden administrativo, lo que unido al retraso en la construcción del edificio definitivo de la escuela de medicina donde funcionaría nuestro laboratorio, motivaron a la dirección de la carrera a realizar una revisión sistemática sobre los nuevos materiales y recursos usados para la enseñanza de anatomía en escuelas de medicina modernas. A partir de este estudio se seleccionaron diferentes materiales innovadores de uso docente para realizar las actividades prácticas. Por otra parte, se remodeló un sector del campus (donde funciona actualmente la carrera) para servir de laboratorio de anatomía. En dicho espacio se ubicaron los nuevos equipos, los que gracias a su versatilidad permitieron su movilización y acomodación según las necesidades de cada actividad práctica en particular. Todo esto permitió subsanar con éxito los inconvenientes antes mencionados, acercándonos además a la metodología docente actual de escuelas de medicina modernas, disminuyendo al mismo tiempo el gasto de recursos que se ocuparían para la mantención de material cadavérico tradicional.

Por otra parte, luego de terminar las actividades de docencia y evaluación de los contenidos de anatomía abarcados durante el primer año de este curso, se aplicó una encuesta para evaluar la percepción de los alumnos acerca del uso de nuevos recursos en la docencia de anatomía. Al observar algunas características de la muestra que fue encuestada, podemos plantear que las notas finales de asignatura y de la parte laboratorio en promedio fueron buenas, con una desviación estándar similar. Este buen rendimiento nos puede orientar a que este primer grupo de alumnos logró integrar las diferentes modalidades de estudio, para conseguir un aprendizaje significativo. Considerando lo anterior, aun cuando no disponemos de datos previos para comparación al ser esta la primera generación de estudiantes de medicina, podemos citar la mejora en rendimiento de los alumnos en Western University of Health Sciences EUA luego de integrar diferentes innovaciones tecnológicas a su asignatura de anatomía, mediante un enfoque combinado (Hasel, 2014).

Acerca de las encuestas recuperadas, podemos decir que se logró una excelente participación de los alumnos con un $100 \%$ de ellos entregando su percepción sobre la docencia recibida. Para la interpretación de los resultados se debe considerar que en cuanto a los géneros que participaron en la encuesta no se observan diferencias importantes, ya que, los alumnos que contestaron se distribuyeron en un $52 \%$ femenino y $48 \%$ masculino.

Al preguntar sobre la utilidad de los fantomas o mo- 
delos anatómicos tanto en su presentación de impreso 3D o cadáver sintético como facilitadores del aprendizaje, se observan porcentajes de aceptación idénticos en ambos recursos $(86 \%)$. Esta percepción de nuestros estudiantes locales tiene concordancia con los resultados presentados en un meta-análisis que incluye 7 trials randomizados, un estudio cuasi-experimental, 820 estudiantes, acerca de la efectividad de los modelos físicos en la enseñanza de anatomía y que describe mejores resultados al usar estos modelos con respecto a otros métodos (Yammine \& Violato, 2016).

Con respecto a la elevada percepción de utilidad de los medios digitales 3D en el aprendizaje, manifestada por el 96,55 \% de los estudiantes encuestados, podemos decir que esta información es concordante con lo reportado en un meta-análisis que incluye 36 estudios de los cuales 28 eran randomizados y basado en 2226 participantes (Yammine \& Violato, 2015). Lo expuesto anteriormente nos hace reflexionar sobre la importante conexión que tienen las actuales generaciones de estudiantes con las nuevas tecnologías y recursos digitales, esta preferencia se ve reflejada en la gran aceptación del conjunto de nuevos recursos 3D (100\% de nuestros encuestados), al compararlos con los tradicionales recursos de aprendizaje 2D, como también se describe en un reporte europeo del uso de imágenes anatómicas 3D en educación médica centrada en el estudiante (Silén et al., 2008). Esta preferencia acerca de las imágenes en 3D también es apreciada en los resultados de los meta-análisis antes citados. Por último debemos mencionar que todos estos nuevos recursos tecnológicos deben utilizarse en forma coordinada e integrada, considerando complementar los recursos de enseñanza tradicionales y fortaleciendo los nuevos enfoques metodológicos basados en casos o problemas, para lograr el diseño eficiente de un curso de anatomía con orientación clínica (Rizzolo et al., 2006).

\section{CONCLUSIONES}

El avance de la tecnología en medicina durante los últimos años ha impulsado el desarrollo de nuevos recursos para la enseñanza de la anatomía, valiéndose de la digitalización de los procesos y materiales usados en docencia, de tal forma que lo aprendido en esta disciplina básica sirva de pilar para las futuras materias clínicas.

Con este trabajo se logra describir por primera vez en nuestro medio local la percepción de los estudiantes de medicina acerca de los nuevos recursos tecnológicos implementados para la enseñanza de la anatomía en nuestra universidad. Al mismo tiempo presentamos datos que servirán de línea base y comparación a próximos estudios sobre los nuevos recursos en docencia. Como resultado de la aplicación de una encuesta de percepción sobre sobre los materiales implementados y su utilidad para el aprendizaje de los contenidos en anatomía, se puede describir una mayor aceptación del uso de materiales innovadores que involucren la visualización de estructuras en 3D versus el uso de recursos tradicionales 2D, ya sea, ocupando un fantoma cadáver sintético, impresiones anatómicas 3D de alta fidelidad o la visualización de reconstrucciones digitales de la anatomía humana mediante programas informáticos. Entre todos estos materiales destaca la gran aceptación de los medios digitales de visualización anatómica 3D. Se debe tener presente que este primer curso de alumnos de medicina corresponde a una generación de estudiantes altamente expuesto a las TIC a lo largo de su vida, factor que debe influir en los procesos de aprendizaje de una nueva disciplina. Esta nueva forma de enseñar anatomía debe motivar a los profesores a capacitarse en el manejo de nuevos materiales y destrezas docentes para desarrollar la asignatura con un enfoque combinado y orientado hacia la clínica. Considerando lo anterior, la aplicación de nuevos recursos tecnológicos en conjunto con la innovación paulatina en metodología docente, nos muestran una mejoría del rendimiento académico promedio en el mismo grupo de estudiantes al finalizar el segundo semestre de la asignatura. Estos resultados de la primera versión del curso deberán ser comparados con el rendimiento de futuras generaciones de estudiantes, además de evaluar la fijación de conocimientos y su persistencia al menos después de un año de aprobada la materia, con el fin de comparar nuestra experiencia con otros trabajos realizados por otras universidades.

HECHT, L. P. \& LARRAZÁBAL, M. A. Use of new technological resources in the teaching of a clinical anatomy course for medicine students. Int. J. Morphol., 36(3):821-828, 2018.

SUMMARY: The use of technological resources as assisting material in the teaching of human anatomy has been referenced in Chile since the 19th century with the first artificial anatomical model. In conjunction with traditional lectures and classical texts on the subject during practical work, the student was presented with a series of strictly anatomical contents that would later be evaluated. This teaching methodology was maintained until well into the 20th century. Subsequently, medical technology progress and innovation was integrated in clinical practice over the last decades. Further, knowledge of anatomical structures, in terms of their representation in exams obtained through medical imaging techniques was also consolidated. For this reason for the education of the new generations of medical doctors, a gradual update was required. These included basic clinical content literature, a teaching methodology with student participation, and use of materials for practical work to achieve learning techniques that would be useful in later courses. Prior to 
the beginning of the school year, the School of Medicine of the Universidad de Tarapacá acquired state of the art material to complement teaching in practical anatomy work for its anatomy laboratory. This article describes implementation of the new technological resources for educational support in anatomy for our school of medicine, among these are: anatomical models printed in three dimensions (3D) Erler-Zimmer ${ }^{\circledR}$, synthetic human cadaver Syndaver $^{\circledR}$, SECTRA $^{\circledR}$ 3D digital dissection table and complementary workstation. All of this material will be used in conjunction with other traditional resources when teaching anatomy, so as to provide an overall view of the contents in this subject area.

KEY WORDS: 3D Print; Anatomical Model; Digital disection; Synthetic cadaver; Medical education.

\section{REFERENCIAS BIBLIOGRÁFICAS}

Cárdenas, V. J. L. The anatomical statue of Dr. Auzoux. First anatomical model of teaching purposes in Chile. Int. J. Morphol., 33(1):393-9, 2015.

Hasel, R. W. Teaching Head \& Neck Anatomy in a Blended Learning Enviroment. In: Teaching with Technology. Pomona, Western University of Health Sciences, 2014. Disponible en: http:// teachtech.westernu.edu/head-neck-anatomy-blended-learning/

Inzunza, O.; Caro, I.; Mondragón, G.; Baeza, F.; Burdiles, A. \& Salgado, G. 3D impressions, new technology that supports anatomical teaching. Int. J. Morphol., 33(3):1176-82, 2015.

Lermanda, S. C. Aprendizaje basado en problemas (ABP): Una experiencia pedagógica en medicina. REXE Rev. Estud. Exp. Educ., (11):12743, 2007.

Montemayor, F. B. G. What the dissection meaning for medical students. Int. J. Morphol., 24(4):575-80, 2006.

Rizzolo, L. J.; Stewart, W. B.; O’Brien, M.; Haims, A.; Rando, W.; Abrahams, J.; Dunne, S.; Wang, S. \& Aden, M. Design principles for developing an efficient clinical anatomy course. Med. Teach., 28(2):142$51,2006$.

Silén, C.; Wirrell, S.; Kvist, J.; Nylander, E. \& Smedby, O. Advanced 3D visualization in student-centred medical education. Med. Teach., 30(5):e115-24, 2008.

Turnis, K. Synthetic Death. Cedar Falls, University of Northern Iowa, 2014. Disponible en: http://www.northerniowan.com/1401/news/syntheticdeath/

Yammine, K. \& Violato, C. A meta-analysis of the educational effectiveness of three-dimensional visualization technologies in teaching anatomy. Anat. Sci. Educ., 8(6):525-38, 2015.

Yammine, K. \& Violato, C. The effectiveness of physical models in teaching anatomy: a meta-analysis of comparative studies. Adv. Health Sci. Educ. Theory Pract., 21(4):883-95, 2016.
Dirección para correspondencia:

Pedro Hecht López

Académico - Laboratorio de Anatomía

Escuela de Medicina - Universidad de Tarapacá

Avda. General Velásquez № 1775

Arica

CHILE

Email: phecht@uta.cl

Recibido : 03-01-2018

Aceptado: 17-04-2018 